\title{
Pacific
}

Journal of

Mathematics

\section{ON A THEOREM OF KOCH}

FARSHID HAJIR 


\title{
ON A THEOREM OF KOCH
}

\author{
FARSHID HAJIR
}

\begin{abstract}
We give a short proof of a slightly stronger version of a theorem of Koch: A complex quadratic field whose ideal class group contains a subgroup of type $(4,4,4)$ possesses an infinite unramified Galois pro-2 extension.
\end{abstract}

\section{Koch's Theorem.}

If $K$ is a finite extension of $\mathbb{Q}$ and $p$ is a prime number, let $K^{(0)}=K$ and for $n \geq 1$ define $K^{(n)}$ to be the maximal abelian unramified $p$-extension of $K^{(n-1)}$. The smallest $n$ such that $K^{(n)}=K^{(n+1)}$ is called the length of the $p$-class field tower of $K$; if no such integer $n$ exists, we say that $K$ has infinite $p$-class field tower. By a group of type $\left(m_{1}, \ldots, m_{t}\right)$ we understand a group isomorphic to $\mathbb{Z} / m_{1} \mathbb{Z} \oplus \cdots \oplus \mathbb{Z} / m_{t} \mathbb{Z}$. The purpose of this note is to give a short proof of (a slightly strengthened version of) a theorem of Koch [4]:

Theorem 1. If $K$ is a complex quadratic field whose ideal class group contains a subgroup of type $(4,4,4)$, then the 2-class field tower of $K$ is infinite.

Koch's proof proceeds by showing that in a minimal presentation of the Galois group of the maximal unramified 2-extension of $K$ by a free pro-2 group $G$, the relations lie deep in the Zassenhaus filtration of $G$. We replace this key ingredient of his proof, which can be thought of as the study of the quadratic unramified extensions of the genus field of $K$ which are central over $K$ [3, Satz 1], with a simple result from genus theory. Moreover, Koch's proof requires a generalization of the Vinberg/Gashütz sharpening of the Golod-Shafarevich theorem on the structure of pro- $p$ groups [4, Satz 3]; for our proof, the original Vinberg/Gashütz inequality suffices (for an account of these inequalities, see, e.g., Koch's book [5]). Indeed, we will need only the following result (see Martinet [8]):

Theorem 2. Suppose $F$ is a totally real field of degree $n$, and $E$ is a totally complex quadratic extension of $F$. Let $t$ be the number of prime ideals of $F$ which ramify in $E$. The 2-rank of the ideal class group of $E$ is at least $t-1$. If

$$
t \geq 3+2 \sqrt{n+1}
$$


then the 2-class field tower of $E$ is infinite.

Corollary 3. Suppose $F$ is a totally real degree 4 extension of $\mathbb{Q}$. If two rational primes that split completely in $F$ ramify in a complex quadratic field $L$, then $E=F L$ has an infinite 2-class field tower.

Proof. With notation as in the theorem, we have $t \geq 8 \geq 3+2 \sqrt{4+1}$.

Proof of Theorem 1. We know that at least four primes divide the discriminant $D$ of $K$. If six or more primes divide $D$, then an application of Theorem 2 to $K / \mathbb{Q}$ already yields the result. Assume first that exaclty four primes divide $D$. By the criterion of Rédei-Reichardt [9] on the 4-rank of the class group of $K$, one knows that $D=-p_{1} \cdot p_{2} \cdot p_{3} \cdot p_{4}$ where $p_{2}, p_{3}, p_{4}$ are odd primes satisfying $\left(\frac{p_{i}}{p_{j}}\right)=+1$ for $i, j>1, i \neq j$, and one of the following is satisfied:

(I) $\quad p_{1}=4 ; p_{j} \equiv 1(\bmod 8), j=2,3,4$.

(II) $p_{1}=8 ; p_{j} \equiv 1(\bmod 8), j=2,3,4$.

(III) $p_{1}=8 ; p_{2} \equiv 7(\bmod 8) ; p_{j} \equiv 1(\bmod 8), j=3,4$.

(IV) $p_{1} \equiv 3(\bmod 4)$ is an odd prime, $p_{j} \equiv 1(\bmod 4), j=2,3,4$, and $\left(\frac{p_{1}}{p_{j}}\right)=+1$ for $j=2,3,4$.

Incidentally, Koch's theorem was originally stated for case (IV) only. Let $F=\mathbb{Q}\left(\sqrt{p_{3}}, \sqrt{p_{4}}\right)$ and $E=F\left(\sqrt{-p_{1} \cdot p_{2}}\right)$. In all cases, $\left(p_{2}\right)$ and the unique rational prime divisior of $\left(p_{1}\right)$ split completely in $F$. Hence, by Corollary 3 , $E$ has an infinite 2-class field tower. Since $E / K$ is an unramified 2-extension, $K$ has an infinite 2-class field tower as well. Now suppose exactly five primes $p_{1}, \ldots, p_{5}$ divide the discriminant of $K$; using the Rédei-Reichardt criterion [9], or its equivalent form due to Rédei [10], it is straightforward to check that for some $i, 1 \leq i \leq 5$, we have

$$
p_{i} \equiv 1 \quad(\bmod 4), \quad\left(\frac{p_{i}}{p_{j}}\right)=1, j \neq i
$$

Now let $F=\mathbb{Q}\left(\sqrt{p_{i}}\right), E=K\left(\sqrt{p_{i}}\right) ; E / F$ is a CM-extension with 8 ramified primes. By Theorem 2, $E$ has an infinite 2-class field tower, and so does $K$.

\section{Further Remarks.}

Koch and Venkov [6] have proved that a complex quadratic field whose ideal class group has a subgroup of type $(p, p, p)$ for some odd prime $p$ has an 
infinite $p$-class field tower. Therefore, a complex quadratic field possesses an infinite Hilbert class field tower whenever its ideal class group contains a subgroup of type $(m, m, m)$ with $m \geq 3$. On the other hand, the field $\mathbb{Q}(\sqrt{-105})$, whose ideal class group is of type $(2,2,2)$, has a finite class field tower, since its root discriminant is just below the Odlyzko bound (see e.g. [8]). I am indebted to the referee for the above remark.

Note that the proof of Koch's theorem we have given relies only on the existence of two primes that split completely in a real biquadratic field. For instance, the primes 31,89 split completely in $\mathbb{Q}(\sqrt{2}, \sqrt{5})$, hence $\mathbb{Q}(\sqrt{-2 \cdot 5 \cdot 31 \cdot 89})$ has an infinite 2-class field tower; its 2-ideal class group is of type $(4,2,2)$.

Taussky-Todd [12] proved that a number field with 2-ideal class group of type $(2,2)$ has a finite 2 -class field tower of length at most 2 . It is natural to ask whether there are number fields with infinite 2-class field tower whose 2class group is of type $(4,2)$ or $(2,2,2)$ (simplest non-cyclic 2-groups after type $(2,2))$. Using a minor variation on an idea first introduced by Schoof [11], we now show that there are complex quadratic fields with these properties. Consider, for example, $K=\mathbb{Q}(\sqrt{-5 \cdot 7 \cdot 41 \cdot 61})$, which has 2-ideal class group of type $(2,2,2)$. To show that this field has infinite 2 -tower, let $H_{0}$ be the Hilbert class field of $K_{0}=\mathbb{Q}(\sqrt{5 \cdot 41 \cdot 61})$, a real quadratic field with class number 16 . Since 7 is inert in $K_{0}$, it splits into 16 prime ideals in $H_{0}$, all of which ramify in the CM extension $L=H_{0}(\sqrt{-7})$. Theorem 2 shows that $L$, an unramified 2-extension of $K$, has an infinite 2-class field tower, proving the claim. In fact, for any prime $q$ satisfying $q \equiv 7(\bmod 5$. $41 \cdot 61$ ) (there are infinitely many such primes by Dirichlet's theorem), the same argument shows that $K_{q}=\mathbb{Q}(\sqrt{-5 \cdot 41 \cdot 61 \cdot q})$ has infinite 2-class field tower; furthermore, by Rédei-Reichardt, $K_{q}$ has 2-class group of type $(2,2,2)$.

For the second example, let $K=\mathbb{Q}(\sqrt{-5 \cdot 11 \cdot 461})$; this field has 2-ideal class group of type $(4,2)$. Observe that the rational prime ideal (11) splits into 16 prime ideals in $H_{0}$, the Hilbert class field of the real quadratic field $K_{0}=\mathbb{Q}(\sqrt{5 \cdot 461})$ with class number 16 . Therefore, by the same argument as above, $L=H_{0}(\sqrt{-11})$, and thereby $K$, have infinite 2-class field tower. Let $H$ be the 2-Hilbert class field of $K$. Benjamin [1] has shown that the 2-class field tower of a complex quadratic field $E$ with 2-class group of type $(4,2)$ has length at most 2 if the 2-Hilbert class field of $E$ has elementary 2-class group $(E=\mathbb{Q}(\sqrt{-5 \cdot 13})$ is an example $)$. Since $K$ has infinite 2tower, we conclude that $H$ does not have elementary abelian 2-class group. 
Finally, note that the 2-rank of the ideal class group of $L$ is at least 15 . Using Louboutin [7], we compute the 2-rank of the ideal class group of the biquadratic field $E=\mathbb{Q}(\sqrt{-11}, \sqrt{5 \cdot 461})$ to be 2 . The arguments of [2] then show that the 2-rank of the ideal class group of $L$ is 15,16 or 17 .

\section{References}

[1] E. Benjamin, Remarks concerning the 2-Hilbert class field of imaginary quadratic number fields, Bull. Austral. Math. Soc., 48 (1993), 379-383.

[2] F. Hajir, On the growth of p-class groups in p-class field towers, J. Algebra, to appear.

[3] H. Koch, Über den 2-Klassenkörperturm eines quadratischen Zahlkörpers. I, J. Reine Angew. Math., 214/215 (1964), 201-206.

[4] _ Zum Satz von Golod-Schafarewitsch, Math. Nachr., 42 (1969), 321-333.

[5] , Galoissche Theorie der p-Erweiterungen, VFB Deutscher Verlag der Wissenschaften, Berlin, 1970.

[6] H. Koch and B. Venkov, Über den p-Klassenkörperturm eines imaginär quadratischen Zahlkörpers, Astérisque, 24/25 (1975), 57-67.

[7] S. Louboutin, Norme relative de l'unité fondamerntale et 2-rang du groupe des classes d'idéaux de certains corps biquadratiques, Acta Arith., 58 (1991), 273-288.

[8] J. Martinet, Tours de corps de classes et estimations de discriminants, Inv. Math., 44 (1978), 65-73.

[9] L. Rédei and H. Reichardt, Die durch vier teilbaren Invarianten der Klassengruppe eines quadratischen Zahlkörpers, J. Reine Angew. Math., 170 (1933), 69-74.

[10] L. Rédei, Arithmetischer Beweis des Satzes über die Anzahl der durch vier teilbaren Invarianten der absoluten Klassengruppe im quadratischen Zahlkörper, J. Reine Angew. Math., 171 (1934), 55-60.

[11] R. Schoof, Infinite class field towers of quadratic fields, J. Reine Angew. Math., 372 (1987), 209-220.

[12] O. Taussky, A remark on the class field tower, J. London Math. Soc., 12 (1937), $82-85$.

Received December 10, 1993 and revised December 7, 1994.

California Institute of Technology

PASADENA CA 91125

E-mail address: fhajir@cco.caltech.edu 


\title{
PACIFIC JOURNAL OF MATHEMATICS
}

\author{
Founded in 1951 by
}

E. F. Beckenbach (1906-1982) $\quad$ F. Wolf (1904-1989)

\section{EDITORS}

Sun-Yung A. Chang (Managing Editor)

University of California

Los Angeles, CA 90095-1555

pacific@math.ucla.edu

F. Michael Christ

University of California

Los Angeles, CA 90095-1555

christ@math.ucla.edu

Nicholas Ercolani

University of Arizona

Tucson, AZ 85721

ercolani@math.arizona.edu
Robert Finn

Stanford University

Stanford, CA 94305

finn@gauss.stanford.edu

Steven Kerckhoff

Stanford University

Stanford, CA 94305

spk@gauss.stanford.edu

Martin Scharlemann

University of California

Santa Barbara, CA 93106

mgscharl@math.ucsb.edu
Gang Tian

Massachusettes Institute of Technology

Cambridge, MA 02139

tian@math.mit.edu

\author{
V. S. Varadarajan \\ University of California \\ Los Angeles, CA 90095-1555 \\ vsv@math.ucla.edu \\ Dan Voiculescu \\ University of California \\ Berkeley, CA 94720 \\ dvv@math.berkeley.edu
}

\section{SUPPORTING INSTITUTIONS}

ACADEMIA SINICA, TAIPEI

CALIF. INST. OF TECHNOLOGY

CHINESE UNIV. OF HONG KONG

HONG KONG UNIV. OF SCI. \& TECH.

KEIO UNIVERSITY

MACQUARIE UNIVERSITY

MATH. SCI. RESEARCH INSTITUTE

NEW MEXICO STATE UNIV.

OREGON STATE UNIV.

PEKING UNIVERSITY

RITSUMEIKAN UNIVERSITY

STANFORD UNIVERSITY

$\begin{array}{ll}\text { TOKYO INSTITUTE OF TECHNOLOGY } & \text { UNIV. OF CALIF., SANTA CRUZ } \\ \text { UNIVERSIDAD DE LOS ANDES } & \text { UNIV. OF HAWAII } \\ \text { UNIV. OF ARIZONA } & \text { UNIV. OF MELBOURNE } \\ \text { UNIV. OF BRITISH COLUMBIA } & \text { UNIV. OF MONTANA } \\ \text { UNIV. OF CALIF., BERKELEY } & \text { UNIV. NACIONAL AUTONOMA DE MEXICO } \\ \text { UNIV. OF CALIF., DAVIS } & \text { UNIV. OF NEVADA, RENO } \\ \text { UNIV. OF CALIF., IRVINE } & \text { UNIV. OF OREGON } \\ \text { UNIV. OF CALIF., LOS ANGELES } & \text { UNIV. OF SOUTHERN CALIFORNIA } \\ \text { UNIV. OF CALIF., RIVERSIDE } & \text { UNIV. OF UTAH } \\ \text { UNIV. OF CALIF., SAN DIEGO } & \text { UNIV. OF WASHINGTON } \\ \text { UNIV. OF CALIF., SANTA BARBARA } & \text { WASHINGTON STATE UNIVERSITY }\end{array}$

The supporting Institutions listed above contribute to the cost of publication of this Journal, but they are not owners or publishers and have no responsibility for its contents or policies.

\section{Manuscripts must be prepared in accordance with the instructions provided on the inside back cover.}

The table of contents and the abstracts of the papers in the current issue, as well as other information about the Pacific Journal of Mathematics, may be found on the Internet at http://www.math.uci.edu/pjm.html.

The Pacific Journal of Mathematics (ISSN 0030-8730) is published monthly except for July and August. Regular subscription rate: $\$ 245.00$ a year (10 issues). Special rate: $\$ 123.00$ a year to individual members of supporting institutions.

Subscriptions, back issues published within the last three years and changes of subscribers address should be sent to Pacific Journal of Mathematics, P.O. Box 4163, Berkeley, CA 94704-0163, U.S.A. Prior back issues are obtainable from Kraus Periodicals Co., Route 100, Millwood, NY 10546.

The Pacific Journal of Mathematics at the University of California, c/o Department of Mathematics, 981 Evans Hall, Berkeley, CA 94720 (ISSN 0030-8730) is published monthly except for July and August. Second-class postage paid at Berkeley, CA 94704, and additional mailing offices. POSTMASTER: send address changes to Pacific Journal of Mathematics, P.O. Box 6143, Berkeley, CA 94704-0163.

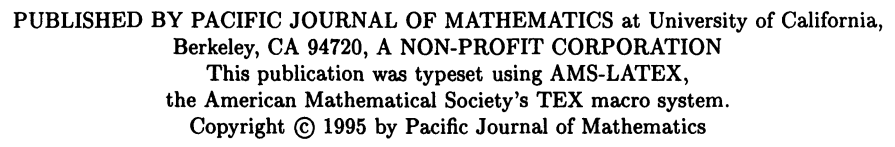




\title{
PACIFIC JOURNAL OF MATHEMATICS
}

\author{
Volume $176 \quad$ No. $1 \quad$ November 1996
}

Moduli spaces of isometric pluriharmonic immersions of Kähler manifolds into

indefinite Euclidean spaces

HitOSHI FURUHATA

On a theorem of Koch

FARSHID HAJIR

Degree-one maps onto lens spaces

Claude Hayat-Legrand, Shicheng WANG and Heiner Zieschang

Unitary representation induced from maximal parabolic subgroups for split $F_{4}$

CHENG CHON Hu

New constructions of models for link invariants

FRANÇOIS JAEGER

Solvability of Dirichlet problems for semilinear elliptic equations on certain domains

ZHIREN JIN

Hadamard-Frankel type theorems for manifolds with partially positive curvature

Katsuei KenMotsu and CHANGYU XIA

Boundary behavior of the Bergman curvature in strictly pseudoconvex polyhedral domains

KANG-TAE KIM and JIYE YU

Existence and behavior of the radial limits of a bounded capillary surface at a corner

KIRK LANCASTER and DAVID SIEGEL

Triangle subgroups of hyperbolic tetrahedral groups

COLIN MACLACHLAN

Chern classes of vector bundles on arithmetic varieties

TOHRU NAKASHIMA and YUICHIRO TAKEDA

Haar measure on $E_{q}(2)$

ARUP KUMAR PAL

Domains of partial attraction in noncommutative probability

VITTORINO PATA

Partitioning products of $\mathscr{P}(\omega) /$ fin

OTMAR SPINAS

Dimensions of nilpotent algebras over fields of prime characteristic

CORA M. STACK

Tensor products of structures with interpolation

FRIEDRICH WEHRUNG

Fourier multipliers for $L_{p}\left(\mathbb{R}^{n}\right)$ via $q$-variation 